\title{
Anisotropy of the Neutron Scattering in the Paramagnetic Phase and on the Spin Waves in the $\mathrm{Mn}_{0.71} \mathrm{Ni}_{0.29}$ Alloy
}

\author{
J. JANKOWSKA-KisielińskA, I. FiJAe-KiREJCZYK \\ AND K. MIKKE \\ Institute of Atomic Energy, 05-400 Otwock-Świerk, Poland
}

\begin{abstract}
We have confirmed the significant anisotropy of the generalised magnetic susceptibility in the paramagnetic and antiferromagnetic phase of the fcc $\mathrm{Mn}-\mathrm{Ni}$ alloys. In this paper the detailed account of the experiments performed on the $\mathrm{Mn}_{0.71} \mathrm{Ni}_{0.29}$ alloy is given. Our main result for the paramagnetic phase is that the correlation length is larger for the direction parallel to the anisotropy axis than for the perpendicular one by a factor of 1.6. The spin-wave velocity observed at $15 \mathrm{~K}$ is higher for the direction parallel to the anisotropy axis than that for the perpendicular direction by a factor of 1.2 . These results are similar to that for the $\mathrm{Mn}_{0.62} \mathrm{Ni}_{0.38}$ alloy. Our results indicate that the magnetic carriers are more localized and the magnetic interactions are less extended in alloys with higher Ni concentration.
\end{abstract}

PACS numbers: 61.05.fm, 78.70.Nx, 75.30.Ds, 75.40.--s, 75.40.Gb

\section{Introduction}

The subject of the present experiment is a test of the spatial anisotropy of the critical neutron scattering in the paramagnetic phase and the scattering on spin-waves $(\mathrm{SW})$ in the antiferromagnetic phase of the $\mathrm{Mn}_{0.71} \mathrm{Ni}_{0.29}$ alloy.

The earlier study of the fcc $\mathrm{Mn}_{0.62} \mathrm{Ni}_{0.38}$ revealed the pronounced uniaxial anisotropy both in the static and dynamical part of the generalised susceptibility $[1,2]$. There are 3 anisotropy axes in the system, each parallel to one of the (100)-type directions, as described in [2]. No significant anisotropy of the SW scattering was found in any other manganese-rich antiferromagnetic alloys with fcc or tetragonal structure. It was observed neither in fcc $\mathrm{Mn}-\mathrm{Fe}$ alloys [3, 4] nor in $\mathrm{Mn}_{0.863} \mathrm{Ni}_{0.137}$ alloy [5]. It was neither mentioned for fcc $\mathrm{Mn}_{0.73} \mathrm{Ni}_{0.27}[6]$. The aim of the present experiment was to test the anisotropy for another $\mathrm{Mn}-\mathrm{Ni}$ alloy with different Mn-content.

Another specific feature of the spin-waves in the fcc $\mathrm{Mn}-\mathrm{Ni}$ alloys is their heavy damping observed in the $\mathrm{Mn}_{0.73} \mathrm{Ni}_{0.27}$ [6] at room temperature and in the $\mathrm{Mn}_{0.62} \mathrm{Ni}_{0.38}[2]$ even at $13 \mathrm{~K}$ (far below $T_{\mathrm{N}}=401 \mathrm{~K}$ ).

\section{Experimental results}

The investigated sample was a single-crystal of dimensions $1 \times 1.2 \times 1.2 \mathrm{~cm}^{3}$. The lattice constant at room temperature is $a=0.370 \mathrm{~nm}$. The crystal mosaic spread FWHM is $\eta=80^{\prime}$. The alloy composition tested by the electron microprobe is: $0.71 \pm 0.01 \mathrm{Mn}$ and $0.29 \pm 0.01 \mathrm{Ni}$. Some amount of $\mathrm{Al}$, below 0.01 , was observed at 5 from 20 tested points.

$\mathrm{Mn}-\mathrm{Ni}$ alloys have a tendency for atomic ordering. Our sample was aged above $800^{\circ} \mathrm{C}$ and quenched to obtain homogeneous alloy. Some short-range order is detected with neutron diffuse scattering.

Below the Néel temperature the alloy is antiferromagnetic of type AF1. The Néel temperature for the investigated sample was obtained from the temperature dependence of the intensity of the elastic neutron scattering at the (100) and (110) reciprocal lattice point - RLP (Fig. 1). The result obtained from the measurement at (100) and confirmed by the measurement at (110) $T_{\mathrm{N}}=408 \pm 1 \mathrm{~K}$ is in agreement to the phase diagram of $\mathrm{Mn}-\mathrm{Ni}$ obtained by the elastic modulus measurement on polycrystal samples [7].

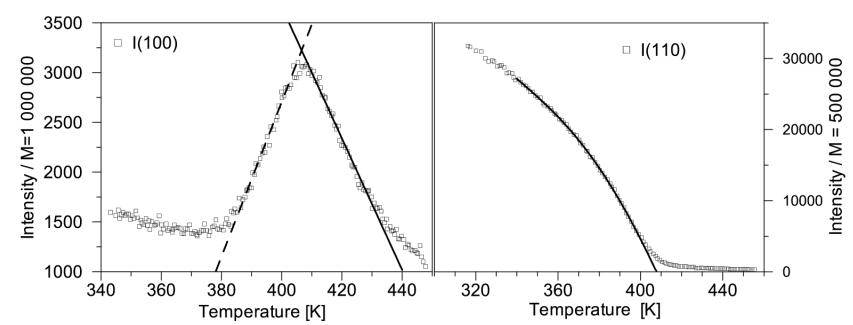

Fig. 1. The temperature dependence of the scattered neutron intensity measured at (100) RLP (left) and at (110) RLP (right).

The spatial anisotropy was tested in the paramagnetic phase for both elastic and inelastic neutron scattering. Elastic scattering was measured at 10, 22, 35 and $45 \mathrm{~K}$ above $T_{\mathrm{N}}$ and inelastic scattering - at 10 and $45 \mathrm{~K}$ above $T_{\mathrm{N}}$. The intensity distributions indicate the strong anisotropy of correlation ranges (Figs. 2, 3).

The experimental data obtained for paramagnetic phase were analysed assuming the neutron-scattering cross-section in the Lorentzian form 


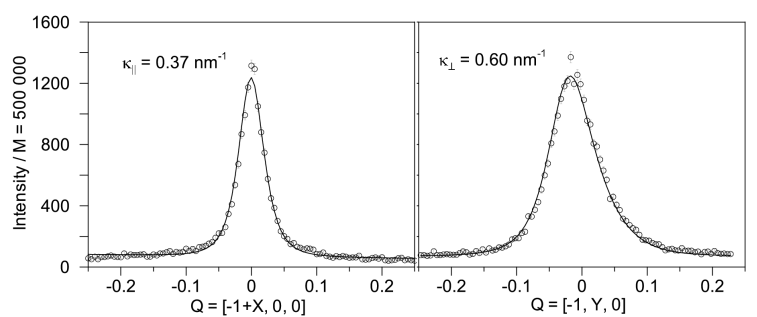

Fig. 2. The distribution of the elastic scattering intensity close to the (100) RLP, versus momentum parallel (left) and perpendicular (right) to the [100] direction, at $418 \mathrm{~K}$.

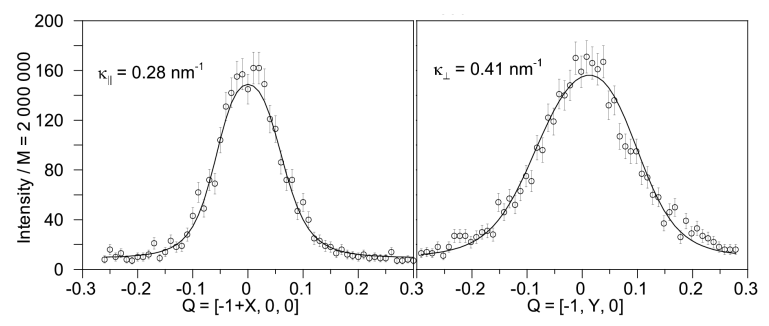

Fig. 3. The inelastic scattering intensity close to the (100) RLP, for the neutron energy loss $5.5 \mathrm{meV}$, versus momentum parallel (left) and perpendicular (right) to the [100] direction, at $418 \mathrm{~K}$.

$$
\begin{aligned}
& \frac{\mathrm{d}^{2} \sigma}{\mathrm{d} \Omega \mathrm{d} E} \propto \frac{E}{1-\exp (-E / k T)} \frac{1}{1+q_{\|}^{2} / \kappa_{\|}^{2}+q_{\perp}^{2} / \kappa_{\perp}^{2}} \\
& \quad \times \frac{\Gamma_{q}}{E^{2}+\Gamma_{q}^{2}},
\end{aligned}
$$

with

$$
\Gamma_{q}=\Gamma_{0}\left(1+q_{\|}^{2} / \kappa_{\|}^{2}+q_{\perp}^{2} / \kappa_{\perp}^{2}\right),
$$

where $E$ is the neutron energy loss, $\boldsymbol{q}$ is the neutron momentum loss reduced to the magnetic Brillouin zone centre of the magnetic structure appearing below $T_{\mathrm{N}} ; q_{\|}, q_{\perp}$, $\kappa_{\|}, \kappa_{\perp}$, are the reduced momentum components and reciprocal correlation lengths for the direction parallel and perpendicular to the anisotropy axis, respectively. $\Gamma_{0}$ is the reciprocal decay time of the fluctuation for $\boldsymbol{q}=0$.

We have found the correlation length for the direction parallel to the anisotropy axis larger than that for the perpendicular direction by a factor of $\kappa_{\perp} / \kappa_{\|}=1.6 \pm 0.1$ for all 4 temperatures. The correlation obtained from inelastic scattering is more extended than the static one, but the ratio $\kappa_{\perp} / \kappa_{\|}$is the same as that obtained from the elastic scattering.

The anisotropy of the neutron scattering on spin waves was tested at $15 \mathrm{~K}$ and $290 \mathrm{~K}$. Constant energy scans were performed in the vicinity of the (100) RLP for $\boldsymbol{q}$ parallel to the [100] and [010] direction and in the vicinity of the (110) RLP (see for an example Fig. 4). The SW energy-gap was obtained from the energy dependence of intensity measured at (100) RLP at $293 \mathrm{~K}$ and $15 \mathrm{~K}$.

The neutron scattering results for spin waves were analysed assuming the cross-section

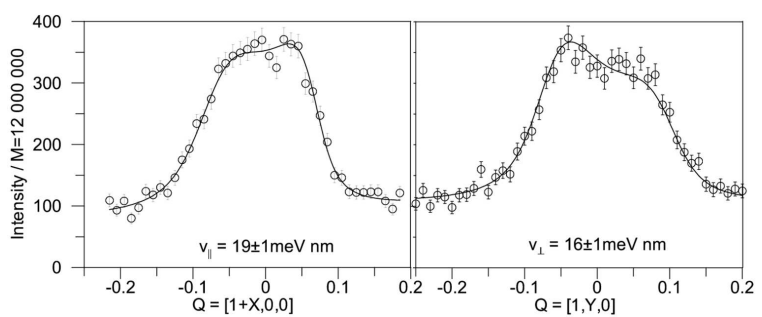

Fig. 4. The inelastic scattering intensity close to the (100) RLP measured at $15 \mathrm{~K}$, for $E=18 \mathrm{meV}$, for $\boldsymbol{q}$ parallel to the [100] direction (left) and to the [010] direction (right).

$$
\begin{aligned}
& \frac{\mathrm{d}^{2} \sigma}{\mathrm{d} \Omega \mathrm{d} E} \propto \frac{E}{1-\exp (-E / k T)} \\
& \quad \times \frac{\Gamma(\boldsymbol{q})}{\left[E^{2}-E(\boldsymbol{q})^{2}\right]^{2}+4 E^{2} \Gamma(\boldsymbol{q})^{2}} .
\end{aligned}
$$

The SW dispersion relation was assumed anisotropic

$$
E(\boldsymbol{q})=E_{\mathrm{g}}^{2}+v_{\|}^{2} q_{\|}^{2}+v_{\perp}^{2} q_{\perp}^{2},
$$

where $E_{\mathrm{g}}=E(\boldsymbol{q}=0), v_{\|}$and $v_{\perp}$ are the SW velocities observed for the direction parallel and perpendicular to the anisotropy axis, respectively. We have assumed the anisotropic damping factor according to

$$
\Gamma(\boldsymbol{q})=\Gamma_{0}+\sqrt{\Gamma_{\|}^{2} q_{\|}^{2}+\Gamma_{\perp}^{2} q_{\perp}^{2}} .
$$

The obtained energy-gap value is $E_{\mathrm{g}}=3.0 \pm 0.04 \mathrm{meV}$ for $293 \mathrm{~K}$ and $E_{\mathrm{g}}=4.4 \pm 0.2 \mathrm{meV}$ for $15 \mathrm{~K}$. The $E_{\mathrm{g}}$ values are similar to those found for $\mathrm{Mn}_{0.62} \mathrm{Ni}_{0.38}[2]$ and $\mathrm{Mn}_{0.73} \mathrm{Ni}_{0.27}[6]$ indicating that the $E_{\mathrm{g}}$ is independent of the alloy concentration in the range $(0.27-0.38 \mathrm{Ni})$, which is in contrast to its strong dependence in the region of the fct-fcc transition $[5,8]$.

The SW velocity obtained from data for $18 \mathrm{meV}$ is: $v_{\|}=19 \pm 1 \mathrm{meV} \mathrm{nm}$ and $v_{\perp}=16 \pm 1 \mathrm{meV} \mathrm{nm}$ for $15 \mathrm{~K}$; and $v_{\|}=14 \pm 1 \mathrm{meV} \mathrm{nm}$ and $v_{\perp}=10 \pm 1 \mathrm{meV} \mathrm{nm} \mathrm{for}$ $293 \mathrm{~K}$. Spin-wave damping factors are $\Gamma_{\|}=5 \pm 2 \mathrm{meV} \mathrm{nm}$ and $\Gamma_{\perp}=5 \pm 1 \mathrm{meV} \mathrm{nm}$ at $15 \mathrm{~K}$ and are small compared to those for $\mathrm{Mn}_{0.62} \mathrm{Ni}_{0.38}$ alloy [2] at this temperature.

\section{Discussion and conclusions}

Our main result for the paramagnetic phase of the $\mathrm{Mn}_{0.71} \mathrm{Ni}_{0.29}$ alloy is that the correlation length is larger for the direction parallel to the anisotropy axis than for the perpendicular one by a factor of $\kappa_{\perp} / \kappa_{\|}=1.6 \pm 0.1$. The SW velocity observed at $15 \mathrm{~K}$ is higher for the direction parallel to the anisotropy axis than that for the perpendicular direction by a factor of $v_{\|} / v_{\perp}=1.2$. The same factors for the $\mathrm{Mn}_{0.62} \mathrm{Ni}_{0.38}$ alloy were: $1.4-1.6$ for the correlation length in the paramagnetic phase and 1.4 for the spin wave velocity at $13 \mathrm{~K}$. Similar anisotropy was found neither in other $\mathrm{Mn}-\mathrm{Ni}$ alloys nor in fcc $\mathrm{Mn}-\mathrm{Fe}$ alloys.

The SW anisotropy is predicted for fcc AF1 antiferromagnetic by the Heisenberg [3, 9] as well as the band model [10]. Within the Heisenberg model the ratio $\kappa_{\perp} / \kappa_{\|}$ 
and the ratio $v_{\|} / v_{\perp}$ should be expressed by the exchange interaction constants for a few nearest neighbours

$$
\begin{aligned}
\frac{\kappa_{\perp}^{2}}{\kappa_{\|}^{2}} & =\frac{v_{\|}^{2}}{v_{\perp}^{2}} \\
& =\frac{-J_{1}+J_{2}+2 J_{3}+4 J_{4}-10 J_{5}+4 J_{6}+\ldots}{J_{2}-4 J_{3}+4 J_{4}+4 J_{6}+\ldots},
\end{aligned}
$$

where $J_{i}$ is the effective exchange interaction between the $i$-th nearest neighbours.

According to Eq. (6) the spatial anisotropy should be expected for all localised AF1-antiferromagnetic fcc systems, and it should be more pronounced for less extended interaction. The most probable reason that spatial anisotropy is not observed in $\mathrm{Mn}-\mathrm{Fe}$ alloys or in $\mathrm{Mn}-\mathrm{Ni}$ with low $\mathrm{Ni}$ concentration, is that their magnetic moments are not so localised as in $\mathrm{Mn}-\mathrm{Ni}$ with $\mathrm{Ni}$ concentration close to $0.3-0.4$. The origin of this difference is rooted in the different electronic structures of both types of alloys. In particular, the calculations of the electron density of states for the Mn-Fe alloys reveal the most itinerant character of magnetic carriers for $\mathrm{Mn}_{0.6} \mathrm{Fe}_{0.4}$ alloys [11]. The density of states for disordered fcc $\mathrm{Mn}_{0.5} \mathrm{Ni}_{0.5}$ alloy [12], with the Fermi energy placed in the region of low density of states, is completely different from that for the Mn-Fe alloys. The dependence of the specific heat coefficient on $\mathrm{Ni}$ concentration [13] supports the results of those calculations. Thus the $\mathrm{Mn}-\mathrm{Ni}$ alloys with high $\mathrm{Ni}$ content have more localised magnetic carriers and less extended magnetic interactions than other fcc Mn alloys.

\section{References}

[1] J.J. Milczarek, K. Mikke, E. Jaworska, J. Phys. (France) 49, C8 (1988).

[2] K. Mikke, J. Jankowska-Kisielińska, B. Hennion, Appl. Phys. A Suppl. 74, S616 (2002).

[3] K. Tajima, Y. Ishikawa, Y. Endoh, J. Noda, J. Phys. Soc. Japan 41, 1195 (1976).

[4] K. Mikke, J. Jankowska-Kisielińska, B. Hennion, Phys. Status Solidi C 3, 151 (2006).

[5] J. Jankowska-Kisielińska, K. Mikke, J.J. Milczarek, J. Phys., Condens. Matter 9, 10761 (1997).

[6] B. Hennion, M.T. Hutchings, R.P. Lowde, M.W. Stringfellow, D. Tocchetti, in: Proc. Int. Conf. on Neutron Scattering, Gatlinburg, Vol. 1, Ed. R.M. Moon, ORNL, Oak Ridge 1976, p. 825.

[7] N. Honda, Y. Tanji, Y. Nakagawa, J. Phys. Soc. Japan 41, 1931 (1976).

[8] J. Jankowska-Kisielińska, K. Mikke, J.J. Milczarek, J. Magn. Magn. Mater. 140, 1973 (1995).

[9] M.W. Long, W. Yeung, J. Phys. C, Solid State Phys. 19, 1409 (1986).

[10] M.W. Long, W. Yeung, J. Phys. F, Met. Phys. 179, 1174 (1987).

[11] S. Asano, J. Yamashita, J. Phys. Soc. Japan 31, 1000 (1971)

[12] J. Yamashita, S. Asano, S. Wakoh, Prog. Theor. Phys. 47, 774 (1972).

[13] W. Proctor, R.G. Scurlock, E.M. Wray, Proc. Phys. Soc. 90, 697 (1967). 